\title{
Anti-PD-1/Anti-CTLA4 DART Protein MGD019
}

National Cancer Institute

\section{Source}

National Cancer Institute. Anti-PD-1/Anti-CTLA4 DART Protein MGD019. NCI Thesaurus. Code C158505.

\begin{abstract}
A hinge stabilized immunoglobulin G4 (IgG4) tetravalent bispecific antibody-like protein directed against the human negative immunoregulatory checkpoint receptors programmed cell death protein 1 (PD-1; PDCD1; CD279) and cytotoxic T-lymphocyteassociated antigen 4 (CT LA4; CT LA-4), with potential immune checkpoint inhibitory and antineoplastic activities. Upon administration, the anti-PD-1/anti-CT LA4 dual-affinity retargeting (DART) protein MGD019 specifically binds to both PD-1 and CT LA4 expressed on tumor-infiltrating lymphocytes (TILS) and inhibits the PD-1- and CT LA4-mediated downregulation of T-cell activation and proliferation. Dual blockade of PD1 and CT LA4 pathways provides enhanced activity against PD1+CT LA4+ double positive cells and may increase T-cell activation and proliferation compared to the blockade of either immune checkpoint alone.
\end{abstract}

